\title{
Minimally Invasive Technique and K-Wire Fixation in Treatment of Fracture Calcaneus M.El-Morsy ${ }^{1}$,H.E.Farag ${ }^{1}$ and H.M.Gomaa ${ }^{2}$ \\ ${ }^{1}$ Orthopaedic Surgery Dept., Faculty of Medicine, Benha Univ., Benha, Egypt \\ ${ }^{2}$ Orthopaedic Surgery Dept., Faculty of Medicine, El-Fayoum Univ., El-Fayoum, Egypt \\ E-Mail: hakemelnaggar@gmail.com
}

\begin{abstract}
The treatment of intra-articular fractures of the calcaneus remains controversial. Different approaches and methods of fixation are used aiming at anatomical reduction and achieving good functional outcome. Patient and methods: 10 patients were included in this study with age range from 20 years to 36 years with mean age 28 years. According to Essex-Lopresti classification, all fractures were joint depression fractures. There were 5 fractures classified as Sanders II , 3 fractures were classified as Sanders III and 2 fractures were classified as Sanders IV. All patients were managed operatively using limited lateral approach and trans-articular k-wires fixation.Results:The mean follow-up duration was 6.20 months. The mean Maryland foot score was 85.50. Complications included pin track infection in 2 cases which were treated conservatively, complex regional pain syndrome in 2 cases, both responded well to physiotherapy, restriction of subtalar joint movement in 3 cases which were treated by physiotherapy. The Sanders classification of fractures was found to be correlated significantly to Maryland foot score.Conclusion : open reduction of displaced intra-articular calcaneal fractures through limited lateral approach and percutneous trans-articular k-wires fixation technique was found to be effective method for treatment of Sanders type II and III , joint depression fractures.
\end{abstract}

Keywords:Intra-articular fractures of the calcaneus, Joint depression type fractures, limited lateral (sinus tarsi) approach, Trans-articular k- wires fixation.

\section{Introduction}

Calcaneal fractures accounts for $2 \%$ of all body fractures and $60 \%$ of tarsal fractures [1].

Axial loading due to fall from height is the commonest cause with $10 \%$ bilateral affection [2].

Calcaneal fractures are mostly inta-articular in adult patients [3].

The articular calcaneal fracture is associated with poor functional outcome making the patient return to day work is difficult and raising the importance of the management [4].

calcaneal fractures have multiple procedures for surgical interventions with different approaches and implants used. Many open surgical approaches have been described including lateral ,medial and combined approaches . open methods are usually copmlicatd with infection and soft tissue damage [5].

Minimally invasive intervention was firstly used by Westhues who introduced the method of closed reduction with percutaneous pinning fixation. This method was later modified and known as Essex-Lopresti technique [6].

The aim of this study is to evaluate the efficacy and the outcomes of surgical treatment of displaced intraarticular fractures of the calcaneus using limited lateral approach and trans-articular k-wires fixation.

\section{Patient and methods}

Ten cases of recent intraarticular fractures of the calcaneus were treated by minimal invasive percutaneous pinning. The patients were operated by multiple surgeons and followed up in Benha university Hospitals \& Fayoum General Hospital during the period between April 2020 to February2021.

All patients in this study were classified according to Essex-Lopresti classification

\section{Evaluation criteria}

Inclusion criteria

Our inclusion criteria include all patients with intraarticular fracture calcaneus, with the following criteria

- Essex-lopresti joint depression type fractures.

- Bad soft tissue condition and open injuries.

\section{Exclusion criteria}

- Old fractures(more than 3 weeks of trauma).

- Extraarticular and tongue type fractures

\section{Perioperative treatment and surgical technique}

Standard lateral and axial views were taken for evaluation of the fracture. CT scan was also done for the affected calcaneus for further evaluation and preoperative planning of the fracture .

The operation was carried out under spinal anaesthesia except one case under general anaesthesia.

All patients were placed on prone position with the foot protruding beyond the edge of the table so that fluoroscopy can be used intraoperatively.

All patients were operated using limited lateral approach (sinus tarsi approach).Straight skin incision is made about $1-2 \mathrm{~cm}$ at the talocalcaneal joint guided by flouroscopy.

The sural nerve and peroneal tendons can be identified and protected by retracting posteriorly .

If there is varus malalignment of the tuberosity fragement, loss of height or length of the calcaneus, a 5 $\mathrm{mm}$ Schanz screw is inserted into the calcaneal tuberosity from medial to lateral. With gentle manual distraction and varus-valgus levering, a closed reduction of the tuberosity is attempted. 
The lateral wall blow out is displaced laterally to get access to the depressed articular fragement(s) of posterior facet. If there is depressed medial articular fragment(s), they should be reduced and fixed before the lateral one. A small elevator is used to disimpact and

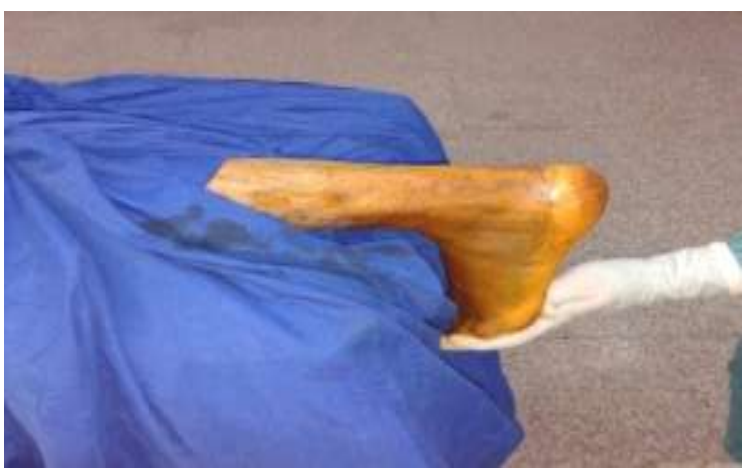

Fig. (1) Intra-operative photo showing position during operation.

Once the reduction is achieved, the articular fragment is fixed using $\mathrm{k}$-wire(s) inserted percutaneously from the sole and passing through the indirectly reduced tuberosity, the reduced articular fragment and the subtalar joint to the talus(trans-articular k-wires fixation).The stability of fixation is checked intraoperatively.

The indirectly reduced tuberosity fragment is fixed using $2 \mathrm{k}$-wires inserted from both sides of achilles tendon insertion posteriorly and passed distally and somewhat laterally to the cuboid.

The lateral wall reduction is achieved and checked by lateral and axial fluoroscopic views.

The wires were bent above the skin level, the wire traction removed and the stab incision closed with rotate the depressed fragment by pulling it back and pushing it underneath the articular surface of the talus. Reduction assessment is checked by the use of flouroscopy.

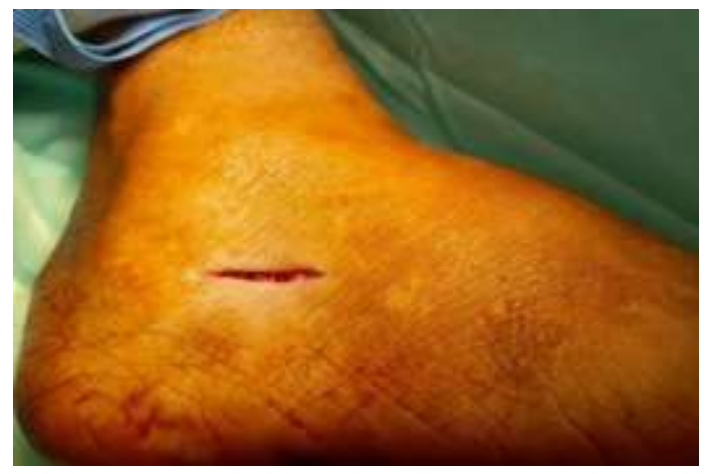

Fig. (2) Intraoperative photo showing skin incision.

sutures, a below knee splint was applied for immobilization and the lower portion of the operative side was elevated.

All patients were followed up at 2,6,8 weeks in the outpatient clinic. Plain $\mathrm{x}$-ray of the calcaneus anteroposterior, lateral and axial views were obtained .At $8^{\text {th }}$ week , the k-wires were removed and the patients were instructed to start subtalar range of motion.

At $12^{\text {th }}$ week the patients were

allowed to start weight bearing after assessment of fracture union both clinically and radiologically. Assessment of the patients by Maryland foot score was done starting from the $6^{\text {th }}$ month postoperatively.
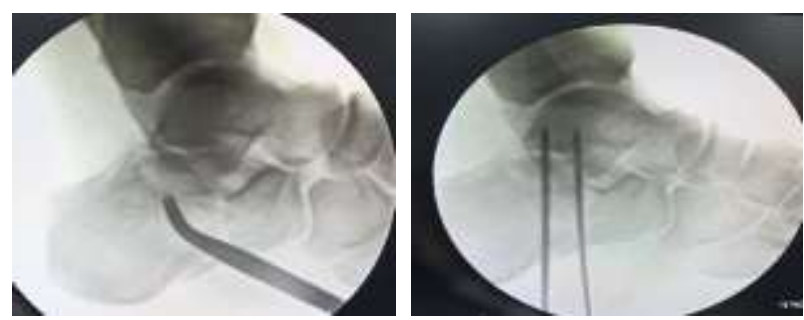

Fig (3) Intraoperative fluoroscopic image showing the posterior articular facet after reduction and trans aritcular k- wire fixation.

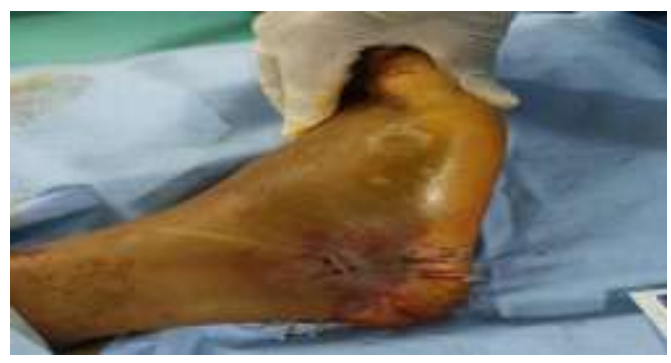

Fig. (5) Intraoperative photo showing the wound after closure.
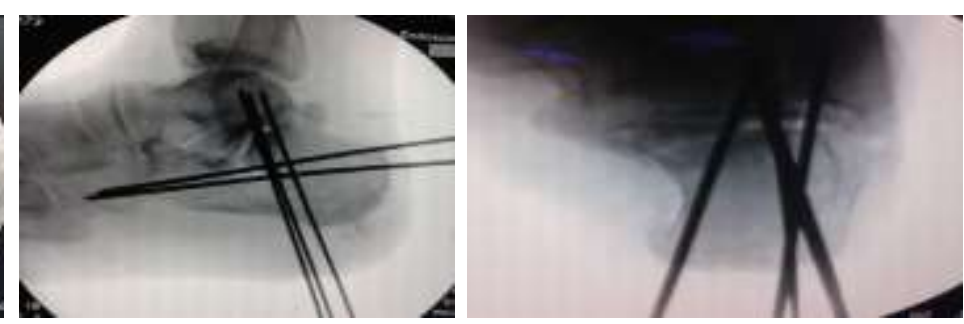

Fig (4) Intraoperative fluoroscopic images showing lateral and axial views of calcaneus after fracture reduction and fixation.
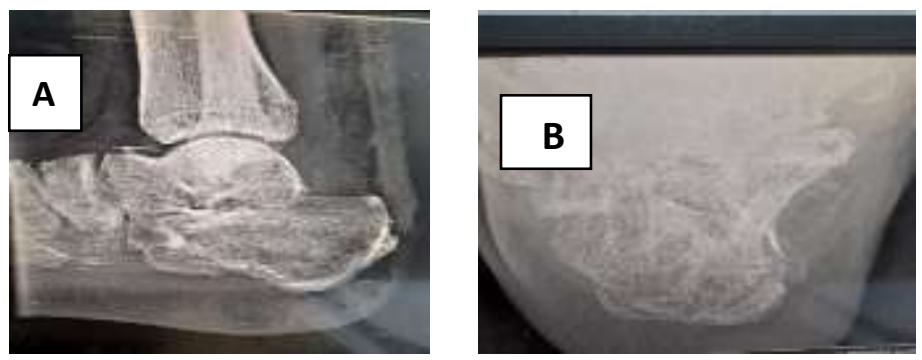

Fig. (6) Plain $x$-rays, lateral (A) and axial(B)views of the calcaneus at the last follow up visit. 


\section{Results}

The average age of our patients was 28 years. The mean follow-up period was 6.20 months. The average union period of fracture was 12 weeks with a range of 10-14 weeks. The average operative time was about 50 min with a range of 45-75 min. Also as regards full WB $90 \%$ show full WB in less than 12 weeks, versus $10 \%$ who show full WB after 12 weeks. As regards time of wire removal $90 \%$ removed it at 8 weeks, versus $10 \%$ who removed it at 10 weeks. The clinical results according to the Maryland foot score revealed one case with fair result (10\%),five cases with good results(50\%) and four cases with excellent results (40\%). Just two patients had superficial pin-tract infections around the Kwires that resolved completely with antibiotics.

\section{Discussion}

Minimally invasive reduction and fixation of calcaneal fractures was developed to minimize complications and correct fracture deformity with good results compared to open techniques [7].

In the current study, the limited sinus tarsi approach was used. This approach was sufficient to expose and reduce the displaced fracture of the posterior facet and the anterolateral fragment under vision. Indirect reduction of the tuberosity fragment was sometime performed.The low incidence of wound problems in this approach may be due to the small skin incision ,minimal soft tissue dissection and more preservation of calcaneal vascularity as compared to the extensile lateral approach.

we presented a minimally invasive technique for the treatment of intraarticular, dislocated calcaneus fractures and were able to produce results comparable to open techniques with a lower rate of serious complications. In the majority of cases, an almost identical Böhler angle and geometry of the calcaneus was achieved when compared to the opposite side at the time of last followup. Simple removal of the Kirschner wires and shorter surgery time decrease patient stress and must be recognized as an advantage of this minimally invasive technique. Thus, we feel that our minimally invasive technique is a viable alternative for the treatment of intraarticular, dislocated calcaneal fractures.

Basile et al. [8] compared 20 patients with extensile lateral approach with 18 patients with minimal invasive sinus tarsi approach the clinical results were similar in both groups . three cases were complicated by wound infection in the first group,while no wound complications occured in the second group. ${ }^{(8)}$

Weber et al. [9] proposed open reduction and internal fixation by plate and screws of displaced intra-articular fractures of the calcaneus for 50 patients. 26 patients were operated upon by the extensile lateral approach and 24 patients were operated upon by limited sinus tarsi approach .The overall clinical results were similar in both groups .However, The wound related complications occurred in $15.4 \%$ of patients in the first group and in $4.2 \%$ of patients of the second group. ${ }^{(9)}$

Holmes et al. [10] stated that the limited sinus tarsi approach offers a minimally invasive incision with excellent exposure to accomplish the goals of fracture reduction and a relatively short operative time that minimizes the risk of soft tissue injury and he had not encountered any wound dehiscence, osteomyelitis, or wound infection. $^{(10)}$

Ragab et al. [11] proposed open reduction through limited sinus tarsi approach and Percutaneous transarticular k-wires fixation for 42 displaced intra-articular fractures of the calcaneus. The results according to calcaneal fracture scoring system were Excellent in 9 patients $(21.4 \%)$, good in 17 patients $(40.5 \%$ ), fair in 8 patients (19\%) and poor in 8 patients $(19 \%)$. There was significant relationship between Sanders classification and the final results. Subtalar arthritis was observed in eight cases $(17.4 \%)$, CRPS occurred in two patients $(4.3 \%)$, Decreased subtalar motion was significant in eight cases $(17.4 \%)$ and mild in the other patients. No cases complicated with infection or wound complication [11].

\section{Conclusion}

Anatomical reduction for intra articular calcaneal fractures is essential for better functional results. Limited lateral approach is an effective approach for reduction of intra articular calcaneal fractures. This approach is sufficient to expose and reduce the displaced posterior facet and anterolateral fragment. As compared to the extensile approach, it has the advantage of being less invasive with less wound healing related complications .

Percutaneous trans-articular k-wires fixation is an efficient method for fixation of joint depression calcaneal fractures. It has the advantages of being less invasive, easier and the absence of problem of pain at the site of implants used for internal fixation that may require second stage operation for removal. However, this method of fixation requires postoperative immobilization for 8 weeks and abolishes the possibility of early range of motion and the limitation of this study.

\section{References}

[1] M.De Filippo , E. Azzali , A.Pesce ,L.Saba , M.Mostardi ,D.Borgia ,et al.

CT arthrography for evaluation of autologous chondrocyte and chondral-inductor scaffold implantation in the osteochondral lesions of the talus.Acta Biomedica Vol.87(3) ,pp. 51-60, 2016.

[2] A.Pinto , A. Reginelli , F. Pinto ,G.Lo Re , F.Midiri , C.Muzj ,et al.Errors in imaging patients in the emergency setting. J Bone Joint Surg. Br.vol. 89,pp.1061, 2016.

[3] C.Piccolo , M.Galluzzo ，S.Ianniello ,M.Trinci , A.Russo , E.Rossi , et al. pediatric musculoskeletal injuries:role of ultrasound and magnetic resonance imaging.Musculoskelet Surg.Vol.101(1) ,pp.85102,2017.

[4] A.Barile , F.Bruno , F.Arrigoni ,A.Splendiani , E.DiCesare , M.Zappia ,et al.Emergency and trauma of the ankle.Semin Musculoskeletal Radio1.Vol.21(3).pp,282-290,2017. 
[5] J.M.Schuberth, M.D.Cobb, R.H.Talarico. Minimally invasive arthroscopic-assisted reduction with percutaneous fixation in the management of intraarticular calcaneal fractures: A review of 24 cases. $\mathbf{J}$ Foot Ankle Surg.vol.48,pp. 315-322, 2009.

[6] S.Rammelt, M.Amlang, S.Barthel, J.M.Gavlik, H.Zwipp. Percutaneous treatment of less severe intraarticular calcaneal fractures. Clin Orthop Relat Res.vol. 468,pp. 983-990, 2010.

[7] N.Kiewiet, B.Sangeorzan . Calcaneal fracture management:extensile lateral approach versus small incision technique. J Foot Ankle Clin. Br.vol. 22(1),pp.77-91, 2017.

[8] A.Basile, F.Albo, A.Via. Comparison between sinus tarsi approach and extensile lateral approach for treatment of closed displaced intra-articular calcaneal fracture. J Foot Ankle Surg Br. Vol. 55,pp. 513-521, 2016.

[9] M.Weber, O.Lehmann, D.Sagesser, F.Krause. Limited open reduction and internal fixation of displaced intra-articular fractures of the calcaneum. J Bone Joint Surg Br. Vol. 90,pp. 1608-16, 2008.

[10] G.B.Holmes Jr,.Treatment of displaced calcaneal fractures using a small sinus tarsi approach. Tech Foot Ankle Surg.Vol.4(1),pp.35-41, 2005.

[11] H.Abdelaleem Ragab, M.Islam. Mubark, M.Ahmed. Nagi,Mohamed Aly Abdelnaby.Treatment of Subtalar Calcanean Fractures Using TransOsseous Limited Lateral Approach. Orthopediia Traumatologia Rehabilitacja MEDSPORTPRESS. Vol. 16(6),pp.629-638, 2014. 